\title{
Diacronie
}

Studi di Storia Contemporanea

$\mathrm{N}^{\circ} 32,4 \mid 2017$

Proiezioni individuali e agire collettivo nella storia

\section{Donne musulmane e lavoro in Medio Oriente e Nord Africa}

\section{Arianna Colella}

\section{Q OpenEdition \\ Journals}

\section{Edizione digitale}

URL: http://journals.openedition.org/diacronie/6827

DOI: 10.4000/diacronie.6827

ISSN: 2038-0925

\section{Editore}

Association culturelle Diacronie

\section{Notizia bibliografica digitale}

Arianna Colella, «Donne musulmane e lavoro in Medio Oriente e Nord Africa », Diacronie [Online], №32, 4 | 2017, documento 9, online dal 29 décembre 2017, consultato il 10 décembre 2020. URL : http:// journals.openedition.org/diacronie/6827 ; DOI : https://doi.org/10.4000/diacronie.6827 


\section{Diacronie}

Studi di Storia Contemporanea

$32,4 / 2017$

Proiezioni individuali e agire collettivo nella storia. Ruoli sociali, aspetti politici e nodi storiografici tra pubblico e privato

\section{Donne musulmane e lavoro in Medio Oriente e Nord Africa}

\section{Arianna COLELLA}

Per citare questo articolo:

COLELLA, Arianna, «Donne musulmane e lavoro in Medio Oriente e Nord Africa», Diacronie. Studi di Storia Contemporanea : Proiezioni individuali e agire collettivo nella storia. Ruoli sociali, aspetti politici e nodi storiografici tra pubblico e privato, 32, 4/2017, 29/12/2017,

URL: < http://www.studistorici.com/2017/12/29/colella_numero_32/ >

Diacronie Studi di Storia Contemporanea $\rightarrow$ http://www.diacronie.it

Rivista storica online. Uscita trimestrale.

redazione.diacronie@hotmail.it

Comitato di direzione: Naor Ben-Yehoyada - João Fábio Bertonha - Christopher Denis-Delacour - Maximiliano Fuentes Codera Anders Granås Kjøstvedt - John Paul Newman - Deborah Paci - Niccolò Pianciola - Spyridon Ploumidis - Wilko Graf Von Hardenberg

Comitato di redazione: Jacopo Bassi - Luca Bufarale - Gianluca Canè - Fausto Pietrancosta - Alessandro Salvador - Matteo Tomasoni - Luca Zuccolo

Diritti: gli articoli di Diacronie. Studi di Storia Contemporanea sono pubblicati sotto licenza Creative Commons 3.0. Possono essere riprodotti e modificati a patto di indicare eventuali modifiche dei contenuti, di riconoscere la paternità dell'opera e di condividerla allo stesso modo. La citazione di estratti è comunque sempre autorizzata, nei limiti previsti dalla legge. 


\title{
9/ Donne musulmane e lavoro in Medio Oriente e Nord Africa
}

\author{
Arianna COLELLA
}

Il lavoro si propone di analizzare il ruolo delle donne nella vita sociale e culturale degli Stati del Medio Oriente e del Nord Africa, in particolare quello che esse assumono nell'ambiente lavorativo. Le donne nel mercato del lavoro sono costrette in una condizione di inferiorità dal momento che le leggi che garantiscono diritti ai lavoratori sono contraddette e violate da leggi di famiglia. Sebbene la loro posizione giuridica sia certamente più penalizzata rispetto a quanto si può constatare nei paesi occidentali, negli ultimi decenni la partecipazione femminile in ambito economico e politico è aumentata. Attraverso lo studio di vari casi è stato dimostrato come la donna abbia assunto un ruolo fondamentale nel mondo arabo-islamico poiché si è compreso che l'emancipazione della donna è una premessa fondamentale per il progresso.

\section{Il contesto socio-culturale}

Nell'immaginario comune del mondo occidentale con l'appellativo di mondo musulmano si è soliti indicare Stati in cui l'Islam è la religione maggiormente diffusa o in cui è religione di Stato. Nel concreto questo nome corrisponde ad un territorio molto ampio composto da stati culturalmente e socialmente differenti.

Le associazioni tra mondo musulmano e movimenti islamici estremisti, governi autoritari e conflitti senza fine, non sono altro che la conseguenza di preconcetti sviluppati nel corso dei decenni a causa di un'errata conoscenza delle dinamiche politiche e sociali degli Stati che ne fanno parte. Sebbene la posizione giuridica delle donne sia certamente più penalizzante rispetto $\mathrm{a}$ quanto si può constatare nei paesi occidentali, negli ultimi decenni sono emerse organizzazioni di donne che mirano a cambiare il diritto di famiglia, che lottano per ottenere uguali diritti tra uomo e donna, che rivendicano leggi contro la violenza domestica e sessuale, che auspicano l'aumento della partecipazione femminile in ambito economico e politico. 
Il ruolo delle donne nella vita sociale e culturale risulta fondamentale per comprendere i profondi cambiamenti in atto nel mondo arabo-islamico ${ }^{1}$ essendo diventata la «liberazione della donna» ${ }^{2}$ una premessa indispensabile per il progresso.

Come precedentemente affermato, parlare di mondo musulmano vuol dire parlare di un'area molto estesa; in questo articolo ne prenderemo in analisi solo una parte, ossia gli Stati che compongono l'area del Medio Oriente e del Nord Africa, differenti per storia e cultura.

Gli Stati che compongono quest'area presentano differenze interregionali per quanto riguarda le norme di genere, per la difesa dello stato legale delle donne, quelle che gestiscono l'accesso all'istruzione, la gestione della fertilità, l'occupazione e la partecipazione politica.

Tutte queste differenze derivano dalla loro eterogeneità politica e dai diversi atteggiamenti culturali legati alle leggi della Shari' $a^{3}$, che sono condizionate dalla cultura di riferimento e dal contesto in cui vengono applicate.

Le norme giuridiche che limitano la libertà della donna hanno subito l'influenza delle politiche economiche e a loro volta sono state causa di una diversificazione economica e sociale nella popolazione. Infatti, negli Stati islamici ci sono delle differenze nello sviluppo delle abilità, nell'industrializzazione, nelle infrastrutture e negli standard di vita, nel sistema di welfare e nella partecipazione femminile alla forza lavoro ${ }^{4}$. Tuttavia, la ricchezza dello Stato non è un fattore determinante nell'emancipazione della donna, come dimostra il caso dell'Arabia Saudita.

Lo sviluppo economico che ha avuto luogo con la fine del colonialismo negli Stati esportatori di petrolio - Arabia Saudita, Oman, Libia, Emirati Arabi, Iran e Iraq - tra il 1969 e il 1980, ha comportato l'inizio del processo urbanizzazione. Il tasso più lento è stato riscontrato in Egitto, la cui popolazione urbana è aumentata solo dal $32 \%$ al $43 \%$ in mezzo secolo ${ }^{5}$. La rapida urbanizzazione avvenuta in queste aree è stata la conseguenza della migrazione dei lavoratori provenienti da altri Stati dell'area, a cui si è aggiunta successivamente la migrazione della manodopera dal Sud e Sud-est asiatico.

\footnotetext{
${ }^{1}$ PEPICELLI, Renata, Femminismo islamico. Corano, diritti, riforme, Roma, Carocci, 2010, p. 9.

${ }^{2}$ Liberazione della donna è il titolo della prima opera di contenuto femminista scritta dall'avvocato egiziano Quasi Amin nel 1899, che sarà poi considerato il paladino della questione femminile nel mondo arabo. PEPICELLI, Renata, op. cit., p. 119.

${ }^{3}$ Shari'a letteralmente "strada/luogo da cui si accede facilmente all'acqua" o "luogo in cui ci si abbevera" sta ad indicare la strada che porta alla salvezza. La Shari'a non è un testo che propaga una serie di norme inequivocabili ma un concetto astratto. Allo stesso tempo è fonte di diritto (figh) che produce norme, anche se non sempre applicabili che corrispondono ad un insieme di corpi testuali che definiscono il rapporto tra uomo e Dio e l'uomo e la società.

${ }^{4}$ MOGHADAM, Valentine M., «Towards gender equality in the Arab/Middle East Region: Islam, culture, and Feminist Activism», in Human Development Report Office Occasional Paper, Illinois State University, 2004, p. 25, URL:

< http://hdr.undp.org/sites/default/files/hdr2004_valentine_moghadam.pdf > [consultato il 19 novembre 2017].

${ }^{5}$ MOGHADAM, Valentine M., «Urbanization and Women's Citizenship in the Middle East», in The Brown journal of World Affairs, XVII, 1/2010, pp. 19-34, p. 19.
} 
Allo sviluppo urbano è seguito lo sviluppo della regione, che ha provocato cambiamenti sociali quali l'aumento della scolarizzazione, la decrescita dei tassi di fertilità, l'aumento dell'età del primo matrimonio e cambiamenti nella struttura familiare. L'urbanizzazione, in concomitanza con l'istruzione, ha permesso un inatteso ritorno all'attivismo politico e al cambiamento culturale. Tuttavia, l'incremento della scolarizzazione non ha eliminato totalmente i problemi riguardanti la qualità dell'istruzione e la pertinenza dell'educazione agli imperativi della globalizzazione; infatti, come per l'economia, la politica e l'urbanizzazione, i livelli di istruzione sono differenti nella regione e la disuguaglianza aumenta tra i centri urbani e le zone rurali, a cui bisogna aggiungere una disuguaglianza di genere. Il tasso di analfabetismo delle donne in tutti i paesi del Medio Oriente e del Nord Africa è molto più alto rispetto a quello degli uomini e raggiunge tassi ancora più alti nelle campagne, nonostante a partire dal 1970 la scolarizzazione sia aumentata considerevolmente in molti paesi ${ }^{6}$.

Negli Stati del Medio Oriente e del Nord Africa il raggiungimento di un uguale - se non maggiore - livello di istruzione non indica un'uguaglianza di genere. Infatti, anche se l'accesso all'istruzione è aumentato, gli esperti fanno notare che vi sono ancora vincoli significativi per le ragazze che vorrebbero completare il ciclo d'istruzione che limitano il loro ingresso nel mercato del lavoro ${ }^{7}$.

Tuttavia, l'urbanizzazione ha comportato anche la crescita delle disuguaglianze, delle tensioni sociali, politiche e culturali e della disoccupazione giovanile.

\section{La condizione giuridica della donna musulmana}

Analizzare il ruolo che la donna ha nelle società del mondo musulmano, in particolare il ruolo che essa assume nell'ambiente lavorativo, non è un obiettivo semplice.

Il concetto di donna musulmana è di per sé un concetto molto vasto che non può essere usato come categoria generalizzata per parlare del ruolo che le donne di religione islamica hanno nelle società del mondo. Non esiste un archetipo di donna musulmana, ma piuttosto donne inserite in differenti gruppi e strutture socio-economiche e culturali. Infatti, un importante fattore di status sociale e giuridico dipende dalle politiche economiche, sociali e culturali vigenti negli Stati in cui esse vivono. A sua volta ciò non implica che all'interno di uno Stato tutte le donne si trovino nella stessa condizione, essendo a loro volta ideologicamente e politicamente divise. Diverse di loro rigettano l'idea della religione e del patriarcato, mentre altre vedono nella religione lo strumento

\footnotetext{
${ }^{6}$ Ibidem, p. 21.

${ }^{7}$ MCLOUGHLI, Claire, Women's economic role in the Middle East and North Africa (MENA) (GSDRC Helpdesk Research Report), Birmingham, Governance and Social Development Resource Centre, University of Birmingham, 2013, pp. 9-10.
} 
per identificarsi come donne. Alcune sono allineate con i liberali o con i socialdemocratici o con le organizzazioni comuniste, altre invece prestano il loro supporto a gruppi islamici e fondamentalisti.

Le donne musulmane costituiscono una diversa ed eterogenea parte della popolazione e il loro ruolo sociale può variare all'interno dello stesso Stato e nei diversi paesi del Medio Oriente e del Nord Africa, a seconda della classe sociale, dell'etnia, dell'età, dell'educazione e del contesto in cui vivono, che siano città o zone rurali. Ciò nonostante, in tutti questi Stati il ruolo che le donne hanno nella società, a differenza di quanto avviene in altri paesi, è prescritto dalla teologia islamica, la quale rappresenta un fattore determinante della legge. Le donne non godono di una cittadinanza piena ma di seconda classe, essendo la loro subordinazione niente altro che la conseguenza dell'applicazione del diritto di famiglia musulmano, il quale deriva da norme patriarcali e culturali ${ }^{8}$ radicate nel tempo.

La forte autorità che la religione islamica ha all'interno della sfera privata, in particolare nella regolamentazione delle dinamiche familiari, ha le sue origini nel periodo post-coloniale. In quegli anni, tra le istituzioni religiose e i capi tribù fu stipulato un compromesso: lo Stato in cambio del pieno potere politico permise alla autorità religiose e tribali di mantenere il controllo sulla regolamentazione dei codici di famiglia.

Il diritto di famiglia musulmano, anche conosciuto come Codice sullo status personale, tratta temi quali il matrimonio, il divorzio, la custodia dei figli e l'eredità. In tutti questi ambiti giuridici le donne godono di meno diritti rispetto agli uomini e in alcuni casi sono addirittura sottoposte alla loro tutela. La condizione delle donne non musulmane sposate con uomini musulmani è anche peggiore, godendo esse di ancor meno diritti ${ }^{9}$.

Una caratteristica dei quadri giuridici degli Stati della regione è la loro inconsistenza. Le garanzie costituzionali di uguaglianza per tutti i cittadini e le leggi che garantiscono diritti ai lavoratori uomini e donne sono contraddette e violate da leggi di famiglia che inducono la donna sotto la tutela di un maschio, dunque in una condizione di inferiorità. Sebbene la legge islamica dia alle donne il diritto di possedere e disporre di beni, queste ereditano meno proprietà rispetto agli uomini. Anche nel caso del contratto matrimoniale la violazione dei diritti si ripete privando le donne della possibilità di esprimere la propria volontà, riducendo il matrimonio ad un accordo tra famiglie piuttosto che tra individui con uguali diritti e obblighi. Inoltre il matrimonio dà al marito il diritto di accesso al corpo della moglie e lo stupro coniugale non è riconosciuto. La moglie è tenuta ad obbedire al marito. I bambini acquisiscono la cittadinanza e lo status religioso

\footnotetext{
${ }^{8}$ MOGHADAM, Valentine M., «Towards gender equality in the Arab/Middle East Region: Islam, culture, and Feminist Activism», cit., p. 29.

${ }^{9}$ Ibidem, p. 36.
} 
solo attraverso i loro padri. Il codice penale prevede l'assoluzione o la riduzione della pena per gli uomini che commettono «delitto d'onore» ${ }^{10}$.

Il diritto di famiglia musulmano scritto secondo le norme della Shari'a è presente in tutti gli Stati, tuttavia esistono delle eccezioni dovute a recenti riforme delle norme, tra queste vi è il caso della Tunisia ${ }^{11}$.

La Tunisia è il paese della regione considerato più liberale, seppure sia governato da una politica autoritaria, che viene preso ad esempio nel campo della legislazione per i diritti delle donne e per l'uguaglianza di genere. Il codice di famiglia tunisino è stato modificato nel 1956 e nel 1968, da allora gli uomini e le donne godono degli stessi diritti ${ }^{12}$. Nel 1991, in supporto alle donne tunisine è stata istituita la commissione "Donne e sviluppo"13, il cui obbiettivo è quello di concentrarsi su questioni riguardanti il genere femminile e permettere loro di diventare partner di sviluppo.

A differenza di quanto accade negli altri Stati, i gruppi di donne sono relativamente influenti in Tunisia. Per giunta, a partire dal 1993 riforme supplementari al diritto di famiglia sono state riconosciute: la promulgazione di queste ha rappresentato una grande vittoria per il movimento ${ }^{14}$.

Sfortunatamente la Tunisia rappresenta la minoranza: infatti, negli altri paesi - come l'Egitto e la Giordania - i movimenti islamisti e i governi conservatori hanno creato un ambiente politicolegale inospitale per le donne, accanendosi sul codice di famiglia e limitando loro la libertà di movimento nelle istituzioni pubbliche ${ }^{15}$.

Ad ogni modo, le Nazioni Unite e le Convenzioni internazionali hanno notato l'emergere di una popolazione di donne istruite e con coscienza politica intenta a ottenere legittimità sociale, giuridica e un cambiamento culturale in favore della parità di genere. Inoltre, molti Stati della regione hanno deciso di prendere parte alle convenzioni internazionali in materia di diritti delle donne aderendo alle linee guida emanate dall'ONU, per migliorarne l'integrazione nello sviluppo e nella partecipazione economica e politica. Fin da subito, tuttavia, è emersa una contraddizione nell'applicazione dei diritti delle donne con le interpretazioni della Shari'a che assumono un peso rilevante nel diritto di famiglia.

\footnotetext{
${ }^{10}$ Ibidem, p. 38.

${ }^{11}$ Ibidem, p. 37.

${ }^{12}$ Ibidem, p. 28.

${ }^{13}$ Ibidem, p. 28.

${ }^{14}$ Ibidem, p. 29.

${ }^{15}$ Ibidem, pp. 36-37.
} 


\section{I movimento femministi islamici}

Oggi il ruolo che la donna musulmana assume nelle società è diventato un tema molto dibattuto negli Stati islamici.

L'Islam non è sempre stata una religione discriminante: nei primi anni della sua diffusione garantì diritti alle donne in misura molto maggiore di altri codici e di altre religioni. Tuttavia, nel corso dei secoli il ruolo che la donna assunse nella società non è stato definito solo da norme religiose e di comportamento stabilite nel Corano, in quanto esse si sono intrecciate ad altri fattori sociali che ne hanno perpetuato lo stato di inferiorità e di disuguaglianza civile e legale.

A questo punto è giusto analizzare il ruolo della donna musulmana solo in relazione all'Islam? E per quanto versi in una condizione di inferiorità, la sua posizione sociale è poi così diversa da quella di donne di altre regioni?

Per trovare delle risposte a queste domande, in primo luogo bisogna indagare il vero ruolo che le donne musulmane hanno nella società di questi paesi allontanandosi, quanto più possibile, dagli stereotipi che influenzano da sempre l'analisi della questione.

Il responsabile della subordinazione della donna musulmana non è di per sé solo l'Islam, in quanto le interpretazioni date al Corano dall'uomo nel corso dei secoli sono poi diventate leggi e imposizioni di precetti religiosi, che si sono radicate nella struttura familiare e nel costume sociale, segnando l'Islam come religione immobile e retrograda. Il ruolo della donna nelle società islamiche non si è adattato alle nuove dinamiche storiche, per arrivare oggi a trovarsi nel mezzo di una duale contraddizione, uguaglianza teologica e disuguaglianza sociale, che ha segnato l'immagine negativa diffusa nella società occidentale.

Tuttavia, la donna non è rimasta in silenzio e in reazione alla sua marginalizzazione, a partire dal XX secolo, ha iniziato un intenso lavoro sociale per la difesa dei diritti, per l'uguaglianza e per la libertà. Indubbiamente la riuscita dei loro obiettivi fu difficile a causa del radicato coinvolgimento della religione nella società, ma ciò non scoraggiò la rivendicazione dei diritti.

La nuova donna musulmana cominciò ad emergere con forza in paesi islamici come l'Egitto, il Libano, la Turchia, la Siria, la Tunisia e il Marocco, ma anche in altri paesi più integralisti come l'Arabia Saudita o l'Iran ${ }^{16}$.

A partire dal $1980^{17}$ con l'intervento di una nuova corrente politica sulla scena pubblica che prese il nome di Rinascita islamica o il più noto fondamentalismo islamico, l'Islam delle origini divenne l'unico tramite per l'emancipazione degli Stati islamici dalla politica coloniale ancora

${ }^{16}$ PÉREZ ÁLVAREZ, Ángeles, REBOLLO ÁVALOS, José, «El Islam en la vida de la mujer a través de los tiempos», in Cauriensia, 4, 2009, pp. 227-247, p. 247.

${ }^{17}$ MOGHADAM, Valentine M., Modernizing Women: Gender and Social Change in the Middle East, Boulder (CO), Rienner, 2013, p. 6. 
presente: nacque così una nuova frangia legata ai movimenti femministi, il Movimento femminista islamico.

Il femminismo islamico, come i movimenti da cui trae origine, vede nella religione islamica lo strumento per la rivendicazione dei diritti delle donne. Alla base della sua ideologia vi è l'idea che i percorsi che portano all'emancipazione femminile non debbano necessariamente svilupparsi adottando il modello universalista dell'ideologia femminista occidentale, ma che possano realizzarsi attraverso l'accettazione e la reinterpretazione critica della propria tradizione e cultura $^{18}$. L'affermazione dei diritti delle donne islamiche non può realizzarsi all'interno di quell'ideologia femminista occidentale che si rifiuta di ascoltare la voce critica delle musulmane e dà per scontato che queste soffrano una condizione di segregazione causata dalla loro fede e che perciò vadano aiutate a liberarsi dall'oppressione della propria religione e cultura ${ }^{19}$.

Il femminismo islamico è un movimento variegato che vede le donne musulmane, arabe e non, rivendicare l'uguaglianza tra i generi partendo dalla reinterpretazione dei testi sacri dell'Islam. Le teologhe e attiviste Fatima Mernissi, Amida Wadud, Asma Barlas fondano la loro critica al patriarcato partendo da una reinterpretazione, la ijtihad ${ }^{20}$, del Corano e degli hadith ${ }^{21}$.

Dal loro punto di vista non bisogna confondere le leggi e i codici attualmente in vigore con il concetto di Shari'a: per loro la volontà divina è eterna, giusta e immodificabile; sono infatti le leggi di derivazione islamica ${ }^{22}$ ad essere patriarcali. Norme che furono scritte esclusivamente da uomini che volontariamente cancellarono il ruolo che le donne ebbero nella storia del primo Islam, durante il quale la religione fu il mezzo che garantì alle donne l'acquisizione di maggiori diritti e il miglioramento delle loro condizioni di vita rispetto al periodo preislamico ${ }^{23}$.

Le femministe islamiche agiscono al di fuori delle tradizionali categorie di «modernità» e di «femminismo»: per loro la religione non deve essere vista come un ostacolo, ma come uno strumento del percorso di liberazione ed emancipazione delle donne. I testi sacri per molte femministe islamiche garantiscono alle donne tutti i diritti: come accedere alla scolarizzazione e al lavoro, come partecipare alla vita politica, come scegliere liberamente il proprio marito e divorziarne se necessario; tutti diritti che oggi le donne si vedono negati o concessi a fatica ${ }^{24}$.

\footnotetext{
${ }^{18}$ PEPICELLI, Renata, Femminismo islamico. Corano, diritti, riforme, cit., p. 22.

${ }^{19}$ Ibidem, p. 26.

${ }^{20}$ Ijtihad, letteralmente "sforzo" è indica la possibilità di più letture e interpretazioni dei testi religiosi. Il ricorso a questa pratica non è più consentito dal X-XI secolo nell'Islam sunnita. In opposizione le femministe islamiche sostengono che la "porta della Ijtihad" per permettere a tutti i fedeli di accedere direttamente alle verità del messaggio divino. Cfr. Ibidem, p. 60.

${ }^{21}$ Hadith sono i detti e i fatti attribuiti al Profeta Mohammad, usati dai fedeli come modello di comportamento. Cfr. Ibidem, p. 9.

${ }^{22}$ MOGHADAM, Valentine M., Modernizing Women: Gender and Social Change in the Middle East, cit., p. 7.

${ }^{23}$ Ibidem, p. 51.

${ }^{24}$ PEPICELLI, Renata, Femminismo islamico. Corano, diritti, riforme, cit., p. 78.
} 
Il femminismo islamico è solo una delle risposte al patriarcato elaborate dal mondo musulmano; se la si considera come unica via si finisce per cadere in quella forma di orientalismo che considera l'Islam come la principale causa della subordinazione femminile e il femminismo come unica strada percorribile per ottenere l'emancipazione ${ }^{25}$. Il movimento delle donne che opera all'interno di una cornice islamica non si sostituisce all'attivismo generale di coloro che agiscono al di fuori dei riferimenti religiosi, ma si affianca a questo contribuendo a diversificare il panorama femminista impegnato nelle lotte per l'uguaglianza di genere del mondo musulmano ${ }^{26}$.

La femminista Asma Lamrabet afferma che la condizione delle donne è nettamente peggiorata negli ultimi secoli. Le cause possono essere ricercate all'esterno (la condizione coloniale) e all'interno (la volontà degli uomini musulmani di escludere le donne dalla sfera pubblica). Le donne, a causa dell'influenza del sistema in cui nascono e crescono, spesso contribuiscono al mantenimento dello status quo, dimostrandosi restie ai cambiamenti. Lamrabet è convinta che in tutto il mondo, sia nei paesi a maggioranza musulmana che in Occidente, sia iniziata una vera e propria rivoluzione silenziosa che, rifacendosi all'Islam delle origini, ridarà alle donne il loro giusto posto nella società ${ }^{27}$.

A partire dal $1990^{28}$ molte femministe rivendicano riforme del diritto di famiglia, considerandone le leggi eredità del tribalismo, di interpretazioni errate e di pregiudizi dei figh ${ }^{29}$ medievali, su cui vennero fondate le scuole giuridiche dell'Islam, chiuse ad ulteriori interpretazioni. I dibatti sulle norme e sulle leggi islamiche riflettono il corso che sta prendendo la Riforma islamica ${ }^{30}$.

\section{La partecipazione femminile al mercato del lavoro}

Negli ultimi anni l'avanzamento dell'urbanizzazione e la possibilità per ampie fasce della popolazione di accedere all'istruzione hanno comportato numerosi cambiamenti sociali e culturali, tra cui la creazione di nuovi posti di lavoro e una maggiore possibilità di accedere alla politica: tali condizioni e cambiamenti sono stati il pretesto per l'emergere di movimenti sociali e l'insorgere di proteste popolari.

L'incremento della scolarizzazione e la possibilità per le donne di accedere all'istruzione ha aiutato le donne a prendere coscienza e consapevolezza del loro ruolo nella società, ciò ha avuto

\footnotetext{
${ }^{25}$ Ibidem, p. 28.

${ }^{26}$ Ibidem, p. 44.

${ }^{27}$ Ibidem, p. 78.

${ }^{28}$ MOGHADAM, Valentine M., «Towards gender equality in the Arab/Middle East Region: Islam, culture, and Feminist Activism», cit., p. 36.

${ }^{29}$ Fiqh è la scienza della giurisprudenza islamica.

${ }^{30}$ MOGHADAM, Valentine M., «Towards gender equality in the Arab/Middle East Region: Islam, culture, and Feminist Activism», cit., p. 37.
} 
anche delle ripercussioni nelle scelte di maternità. La crescita dei livelli di urbanizzazione e di istruzione ha provocato la diminuzione del tasso di fertilità delle donne musulmane. Infatti in questa area, che fino a poco tempo fa aveva tra i più alti tassi di crescita del mondo, è avvenuta una decrescita demografica, soprattutto tra le giovani donne istruite delle città. Tuttavia il comportamento delle donne legate al mondo agricolo rispetto alla fertilità è abbastanza diverso da quello delle donne inserite nel contesto urbano.

Come affermato dal demografo John Caldwell, la presa di coscienza delle donne sulla loro funzione riproduttiva ha implicato una transizione verso una maggiore apertura al lavoro e ad altre attività sociali: ciò potrebbe riflettere il calo delle forti opinioni morali suoi ruoli distinti dei sessi e sulla sacralità della maternità ${ }^{31}$.

Gli standard di vita nella regione hanno subito dei miglioramenti: la rapida urbanizzazione e la crescita della popolazione hanno cambiato la struttura della forza lavoro, coinvolgendo le donne, anche se in proporzioni inferiori rispetto ad altre regioni. I dati dell'Organizzazione Internazionale del Lavoro (ILO) ${ }^{32}$ mostrano che il tasso di attività economica delle donne adulte non supera il $32 \%{ }^{33}$.

Il World Development Indicators ${ }^{34}$ del 2012 ha evidenziato come le donne del Medio Oriente e del Nord Africa abbiano fatto numerosi passi avanti nell'acquisizione di diritti nel campo della salute e dell'istruzione.

Tuttavia, i dati dimostrano che l'aumento del livello di istruzione non si riflette sulla partecipazione femminile alla forza lavoro. Questa situazione, è descritta dalla Banca Mondiale come il paradosso del Medio Oriente e del Nord Africa ${ }^{35}$, che viene spiegato come il principale risultato dei cambiamenti nella struttura economica e nell'offerta del mercato del lavoro.

Il lavoro nei paesi della regione è caratterizzato dalla segmentazione del mercato: ciò sta ad indicare la tendenza di uomini e donne a non occupare gli stessi impieghi. I livelli di partecipazione femminile al lavoro sono molto alti nel settore pubblico: le donne istruite che

\footnotetext{
${ }^{31}$ MOGHADAM, Valentine M., «Urbanization and Women's Citizenship in the Middle East», cit., p. 23.

${ }^{32}$ L'Organizzazione Internazionale del Lavoro (ILO) è l'agenzia delle Nazioni Unite che si occupa di promuovere il lavoro dignitoso e produttivo in condizioni di libertà, uguaglianza, sicurezza e dignità umana per uomini e donne, URL: < http://ilo.org/rome/ilo-cosa-fa/lang--it/index.htm > [consultato il 19 novembre 2017].

${ }^{33}$ INTERNATIONAL LABOUR OFFICE, «Discrimination at Work in the Middle East and North Africa», in Declaration on Fundamental Principles and Rights at Work, p. 1, URL:

<http://www.ilo.org/wcmsp5/groups/public/---ed_norm/--

declaration/documents/publication/wcms_decl_fs_92_en.pdf > [consultato il 19 novembre 2017].

${ }^{34}$ La Banca Mondiale è la principale raccolta di indicatori di sviluppo, compilata da fonti internazionali ufficialmente riconosciute. Presenta i più attuali e accurati dati di sviluppo e include stime nazionali, regionali e globali, URL: < http://data.worldbank.org/data-catalog/world-development-indicators > [consultato il 19 novembre 2017].

${ }_{35}$ MCLOUGHLI, Claire, Women's economic role in the Middle East and North Africa (MENA) (GSDRC Helpdesk Research Report), cit., p. 9.
} 
vivono in città tendono a concentrarsi in pochi settori occupazionali, in particolare nel settore dei servizi ${ }^{36}$, in quanto offre migliori opportunità di bilanciamento vita-lavoro.

La concentrazione dell'occupazione femminile nel settore pubblico e la loro mobilità limitata sono considerati i fattori chiave alla base degli alti livelli di disoccupazione nella regione. Il lavoro femminile è inoltre scoraggiato dalla mancanza di trasporto sicuro e affidabile che costituisce un vincolo pratico e, di conseguenza, le donne tendono a lavorare all'interno della propria area di residenza o direttamente in casa.

La limitata presenza di forza lavoro femminile nel lavoro privato corrisponde alla quasi totale mancanza di protezioni e di leggi di tutela in questo settore, nel quale le donne sono sottoposte a un alto numero di ostacoli. Per esempio le imprenditrici devono affrontare numerosi problemi per accedere a prestiti e finanziamenti, a causa delle politiche creditizie conservatrici secondo le quali non è opportuno concedere prestiti a donne. In assenza di finanza formale la maggior parte delle donne imprenditrici fa affidamento a fonti private per finanziare le loro attività e ciò costituisce un possibile ostacolo per la crescita a lungo termine delle economie statali.

La mancanza di tutele nel settore privato ha trasformato le donne in "forza lavoro vulnerabile", ossia più disponibili degli uomini a lavorare nel settore informale ${ }^{37}$ (nel lavoro temporaneo, stagionale, agricolo o come badanti), per integrare le carenze di reddito familiare.

Il fattore che maggiormente vincola la partecipazione femminile alla forza lavoro è la loro incapacità, o più propriamente la loro impossibilità, di continuare a lavorare nel settore privato dopo il matrimonio: diventa infatti difficile per le donne conciliare il lavoro nel settore privato con le responsabilità familiari di cui devono farsi carico. Secondo alcuni osservatori una soluzione al problema sarebbe trovare un modo per ridurre il costo per i datori di lavoro privati per assumere donne sposate, di norma impegnate nella cura dei figli e in altre responsabilità ${ }^{38}$.

Gli stereotipi culturali che inquadrano le donne in un ruolo famigliare e gli uomini come principale fonte degli introiti, sono ritenuti gli ostacoli più impedenti per la partecipazione femminile allo sviluppo economico della regione.

In molti paesi il tasso di disoccupazione femminile è rimasto più alto rispetto a quello maschile, nonostante i più bassi livelli di partecipazione alla forza lavoro. Questi dati dimostrano la preferenza delle donne a lavorare nel settore pubblico, che garantisce minori posti di lavoro rispetto a quello privato e che è molto più discriminatorio. La disoccupazione femminile è più elevata in tutto il mondo, ma il divario tra uomo e donna è particolarmente alto nelle regioni del Medio Oriente e del Nord Africa: è infatti il 25\% maggiore di quella maschile, che nel 1990 era al

\footnotetext{
${ }^{36}$ Ibidem, p. 7.

${ }^{37}$ Con settore informale si indicano tutti i lavori non tutelati dalla legge. Cfr. Ibidem, p. 7.

${ }^{38}$ Ibidem, p. 9.
} 
10\% in Algeria, Tunisia, Egitto, Giordania, Iran, Turchia e Yemen ${ }^{39}$; ciò evidenzia una crescita delle donne in cerca di lavoro in funzione sia del livello di istruzione che del bisogno economico.

In tutti i paesi della regione le donne sono particolarmente vulnerabili alla povertà durante i periodi di difficoltà economica e in caso di divorzio, abbandono e vedovanza. Questa vulnerabilità può essere la conseguenza di differenze nell'alfabetizzazione, nel raggiungimento dell'educazione, nella disoccupazione, ma soprattutto dell'esasperazione delle norme culturali che identificano l'uomo come capofamiglia e la donna come casalinga ideale. Il diritto di famiglia musulmano discrimina le donne in materia di eredità e incoraggia la dipendenza dall'uomo "guardiano" della famiglia. La connessione tra l'alto tasso di disoccupazione e la condizione della donna nella cultura musulmana è presa in considerazione dalle sostenitrici della riforma del diritto ${ }^{40}$.

Nel 2012 la Commissione economica e sociale per l'Asia Occidentale ${ }^{41}$ ha dimostrato che le carenze nella legislazione e in particolar modo l'assenza di misure di regolamentazione hanno ostacolato l'accesso delle donne al mondo del lavoro ${ }^{42}$.

Uno studio sui diritti ha rivelato che in quasi tutti i paesi del Medio Oriente e del Nord Africa, le leggi sul lavoro proibiscono alle donne di intraprendere qualsiasi tipo di lavoro ritenuto pericoloso, arduo o dannoso per la loro salute e morale, impedendone in alcuni casi la partecipazione a settori importanti dell'economia. Numerosi Stati hanno leggi che vietano alle donne di lavorare di notte e quando si ritiene che sia pericoloso per loro viaggiare. Queste restrizioni mirano a limitare le opzioni di lavoro e la mobilità. Per esempio alle donne è vietato lavorare nei panifici o in negozi con orari notturni ${ }^{43}$.

La Banca Mondiale ${ }^{44}$ sostiene che questi tipi di legislazioni abbiano come obiettivo quello di dare protezione sociale alle donne, non esponendole a condizioni pericolose di lavoro, ma allo stesso tempo sono discriminatorie ed hanno essenzialmente lo scopo di evitare conflitti con le pratiche culturali tradizionali.

Gli esperti sostengono che, anche se le leggi di parità sono state varate nella maggior parte dei paesi, le pratiche consuetudinarie possono ancora impedire alle donne di esercitare i loro diritti legali. Anche dove le leggi sono emanate per sostenere i diritti economici delle donne, la loro

\footnotetext{
${ }^{39}$ MOGHADAM, Valentine M., «Urbanization and Women's Citizenship in the Middle East», cit., p. 26.

${ }^{40}$ Ibidem, p. 27.

${ }^{41}$ La Commissione economica per l'Asia occidentale (ECWA) è stata istituita il 9 agosto 1973. Il suo scopo era quello di stimolare l'attività economica nei paesi membri, rafforzare la cooperazione tra loro e promuovere lo sviluppo. Nel 1985 prese il nome di Commissione economica e sociale per l'Asia occidentale (ESCWA), URL: < https://www.unescwa.org/about-escwa > [consultato il 19 novembre 2017].

${ }^{42}$ MCLOUGHLI, Claire, Women's economic role in the Middle East and North Africa (MENA) (GSDRC Helpdesk Research Report), cit., p. 9.

${ }^{43} \mathrm{Ibidem}, \mathrm{p} .12$.

${ }^{44}$ La Banca Mondiale è una delle maggiori fonti di finanziamento e conoscenza del mondo per i paesi in via di sviluppo. Le sue cinque istituzioni condividono l'impegno per ridurre la povertà, aumentare la prosperità condivisa e promuovere lo sviluppo sostenibile, URL: < http://www.worldbank.org/en/who-we-are > [consultato il 19 novembre 2017].
} 
partecipazione dipende dalla scuola teologica prevalente nel paese. Infatti, la presenza nella regione di numerose sette musulmane che rispettano le leggi di successione della Shari'a crea dei contrasti con le leggi statali, che garantirebbero parità di diritti ${ }^{45}$.

Le leggi per la parità di retribuzione tra uomo e donna per lo stesso tipo di lavoro e le pari opportunità di formazione e di promozione sono spesso violate, perché gli enti governativi non hanno la capacità di indagare i casi di discriminazione o di imporre sanzioni per tali violazioni, in particolare per quanto riguarda il congedo di maternità e le leggi di eredità. Inoltre, nel sistema giuridico non esistono leggi che proibiscano le molestie sessuali e che ne puniscano i colpevoli ${ }^{46}$.

Il congedo di maternità è considerato inadeguato in quasi tutti i paesi del Medio Oriente e del Nord Africa. Le ricerche condotte dall'ESCWA dimostrano che gli unici paesi a fornire un adeguato pagamento durante il congedo di maternità sono l'Egitto (3 mesi), il Marocco (14 settimane) e la Repubblica araba siriana (120 giorni). L'assenza di adeguati congedi di maternità in parte è dovuta al fatto che non sono i governi a pagarne i costi, che invece ricadono sul datore di lavoro. Questo meccanismo può agire come disincentivo per i datori di lavoro del settore privato ad impiegare donne in età fertile ${ }^{47}$.

Le leggi sull'eredità che favoriscono l'uomo lasciano, come affermato, poco spazio alle donne per ottenere prestiti, per avviare o far crescere le loro aziende.

In una regione così diversa in termini sociali, culturali e religiosi, numerose sono le forme di discriminazione che si basano sulle origini sociali, religiose ed etniche. Forme discriminatorie che ricalcano l'esperienza ghettizzante che i musulmani, uomini e donne, subiscono al di fuori della loro regione di origine. Il fattore di maggiore discriminazione è la religione, essendo la libertà religiosa inesistente ${ }^{48}$.

Gli Stati della regione sono origine e destinazione di migranti provenienti dall'area asiatica, che lavorano principalmente nel campo delle costruzioni, del lavoro domestico e dell'agricoltura. La condizione in cui versano le donne migranti è peggiore di quella delle donne musulmane, avendo maggiori difficoltà di entrare nella forza lavoro legale. Il lavoro delle donne migranti è concentrato in pochi settori definiti che le rendono molto vulnerabili allo sfruttamento rispetto ai migranti uomini, per esempio nell'agricoltura, nell'industria del sesso e nel lavoro domestico.

Negli Stati del Golfo le donne migranti rappresentano il 20-40\% della forza lavoro e molte di loro provengono dal Sud e dal Sud-est asiatico ${ }^{49}$. Sebbene le loro condizioni di lavoro possano variare enormemente, queste lavoratrici sono particolarmente soggette a discriminazioni,

\footnotetext{
${ }^{45}$ MCLOUGHLI, Claire, Women's economic role in the Middle East and North Africa (MENA) (GSDRC Helpdesk Research Report), cit., p. 13.

${ }^{46}$ Ibidem, p. 14.

${ }^{47}$ MCLOUGHLI, Claire, Women's economic role in the Middle East and North Africa (MENA) (GSDRC Helpdesk Research Report), cit., p. 12.

${ }^{48}$ INTERNATIONAL LABOUR OFFICE, «Discrimination at Work in the Middle East and North Africa», cit., p. 3.

${ }^{49}$ Ibidem, p. 2.
} 
sfruttamento, molestie e violenze da parte dei datori di lavoro, coercizione da parte delle agenzie di lavoro, lavoro forzato, bassi salari e inadeguate coperture sociali. Inoltre la discriminazione salariale è collegata alla nazionalità: per esempio i lavoratori provenienti dalle Filippine ricevono salari relativamente alti rispetto a lavoratori provenienti dall'Indonesia e dallo Sri Lanka.

Per comprendere quanto le norme culturali e sociali influiscano sulle scelte lavorative delle donne musulmane è interessante analizzare le analogie tra la partecipazione alla forza lavoro delle donne native del Medio Oriente e del Nord Africa e le donne provenienti dalla stessa area geografica che vivono in Europa. È evidente come le donne provenienti da società conservatrici tendano in misura minore a partecipare al mercato del lavoro rispetto a nativi o a immigrati provenienti da paesi con un alto tasso di occupazione femminile ${ }^{50}$. Dagli studi portati avanti nei Paesi Bassi è emersa una maggiore probabilità tra le donne di provenienza turca e marocchina, con un livello di educazione inferiore al limite stabilito dalla legge, a non far parte della forza lavoro $^{51}$.

Pertanto, per gli Stati europei comprendere l'influenza che può avere la cultura nella partecipazione femminile alla forza lavoro è fondamentale, dato l'alto numero di immigrati provenienti da paesi maggioritariamente musulmani. Una maggiore attenzione ai fattori culturali può incentivare futuri interventi politici indirizzati a facilitare l'entrata di molte donne nel mondo del lavoro ${ }^{52}$.

Gli immigrati sono una risorsa sempre più importante nei paesi europei per la forza lavoro che offrono a medio e lungo termine, alla luce del rallentamento della crescita della popolazione e dell'invecchiamento della generazione del baby-boom. Le donne del Sud del Mediterraneo e del Medio Oriente costituiscono il gruppo con il maggior potenziale economico per l'Europa ${ }^{53}$.

L'istruzione ha un ruolo imprescindibile per l'emancipazione delle donne e per il loro inserimento nel mondo del lavoro ${ }^{54}$.

\footnotetext{
${ }^{50}$ NADEREH, Chamlou, MUZI, Silvia, HANANE, Ahmed, «Understanding the Determinants of Female Labor Force Participation in the Middle East and North Africa Region: The Role of Education and Social Norms in Amman», in Almalaurea Working Papers, 31, 2011, pp. 1-19, p. 1.

${ }^{51}$ Ibidem, p. 1.

${ }^{52}$ Ibidem, p. 2.

${ }^{53}$ Ibidem, p. 2.

${ }^{54}$ Ibidem, p. 2.
} 


\section{Il ruolo della donna nel futuro economico del Medio Oriente e del Nord} Africa

Negli Stati del Medio Oriente e del Nord Africa la partecipazione delle donne al mercato del lavoro è in pieno sviluppo, ma il loro ruolo nella società continua ad essere fortemente condizionato dalle norme culturali e sociali vigenti. Nonostante siano stati fatti numerosi passi avanti per migliorare le condizioni delle donne, negli ultimi anni, poche sono state le iniziative di governo che hanno affrontato direttamente i vincoli culturali e facilitato la mobilità delle donne nel mercato del lavoro.

L'inserimento nel mercato del lavoro dovrà avvenire evitando discriminazioni di genere e, come avverte la Commissione economica e sociale per l'Asia occidentale, se le politiche del lavoro volte a responsabilizzare le donne possono riuscire a creare maggiori opportunità, allo stesso tempo, possono involontariamente rafforzare gli stereotipi di genere55. La creazione di posti di lavoro esclusivi potrebbe infatti rafforzare la convinzione negativa che le donne non possono far parte della forza lavoro tradizionale. Tali misure contraddirebbero le politiche per l'occupazione dell'ILO56 sui diritti dei lavoratori e sulla libertà di scelta in materia di occupazione. L'ESCWA sostiene una soluzione basata sulla revisione dei diritti, un esempio potrebbe essere cercare di ridurre il divario tra gli stipendi di uomini e donne che permetterebbe di sviluppare l'idea che le donne non siano inferiori all'uomo.

La Giordania è lo Stato che negli ultimi anni ha applicato maggiori riforme per permettere la crescita dell'occupazione femminile. In questo caso il Ministero del Lavoro in collaborazione con l'Organizzazione Internazionale del Lavoro ha reato un comitato direttivo nazionale per la parità salariale ${ }^{57}$. Ulteriori riforme sono state apportate per assicurare la maternità retribuita e per risolvere il grave problema che porta i datori di lavoro del settore privato a non assumere donne in età fertile. In base alle nuove disposizioni, datori di lavoro e dipendenti contribuiscono ad un "Fondo di maternità" devolvendo lo 0,75 \% di ogni busta paga. Questo dovrebbe aumentare gli incentivi ad assumere le donne, ad incoraggiare la loro partecipazione al mercato del lavoro e limitarne la discriminazione.

\footnotetext{
${ }^{55}$ MCLOUGHLI, Claire, Women's economic role in the Middle East and North Africa (MENA) (GSDRC Helpdesk Research Report), cit., p. 17.

${ }^{56}$ L'ILO è l'unica agenzia tripartita delle Nazioni Unite che riunisce datori di lavoro e lavoratori di 187 Stati membri, per stabilire norme di lavoro, sviluppare politiche e ideare programmi che promuovano un lavoro dignitoso per tutte le donne e gli uomini, URL: <http://www.ilo.org/global/about-the-ilo/lang-en/index.htm> [consultato il 19 novembre 2017].

${ }^{57}$ MCLOUGHLI, Claire, Women's economic role in the Middle East and North Africa (MENA) (GSDRC Helpdesk Research Report), cit., p. 17.
} 
La Giordania non è l'unico Stato della regione ad aver attuato politiche per il miglioramento della condizione femminile nella società, infatti in altri paesi vi sono diversi esempi di buone iniziative volte a promuovere l'attività imprenditoriale delle donne.

Il Centro Business Development delle donne in Egitto ${ }^{58}$ è considerato il precursore nella regione ed è un esempio di buona pratica nel fornire sostegno alle piccole imprese in via di sviluppo. La Casa Pionnieres, in Marocco ha avuto successo nel fornire supporto tecnico e finanziario alle imprese femminili. Tra il 2006 e il 2010 ha contribuito allo sviluppo di 50 aziende in settori diversi, tra cui il turismo, la formazione e la cura dei bambini59. E infine la palestinese Associazione delle Imprenditrici (ASALA) fornisce servizi di sviluppo aziendale per donne microimprenditrici nella striscia di Gaza e in Cisgiordania ${ }^{60}$.

Come emerge dalle ultime ricerche il basso livello di partecipazione delle donne alla forza lavoro non dipende dal basso reddito pro-capite, dagli elevati tassi di analfabetismo o dai bassi livelli di urbanizzazione, ma piuttosto dalle retrograde norme culturali e sociali.

Prendendo in esame il caso dell'Egitto emerge che l'alta disoccupazione femminile è legata alle interpretazioni ortodosse del Corano e ai codici di famiglia patriarcali61. Infatti, l'atteggiamento degli uomini nella famiglia diventa una barriera che non permette alle donne di impegnarsi nella ricerca di un lavoro al di fuori delle mura domestiche.

Per migliorare l'accesso delle donne alla forza lavoro bisognerebbe costruire un quadro giuridico e normativo che le svincoli dal controllo dell'uomo. Impegnarsi nel far cambiare gli atteggiamenti degli uomini conservatori, giovani in particolare, nei confronti delle donne che lavorano fuori casa potrebbe contribuire ad aumentare i tassi di partecipazione femminile alla forza lavoro. La riforma dell'istruzione e l'abolizione di preconcetti culturali che identificano la donna come moglie e madre dovrebbe essere per i governi il primo punto su cui intervenire. L'intervento legislativo garantirebbe loro maggiore libertà di mobilità, maggiore sicurezza nei luoghi di lavoro e più possibilità di partecipare all'imprenditoria.

Le donne musulmane diventeranno cittadine e portatrici di tutti i diritti solo dopo che gli Stati a maggioranza islamica attueranno dei cambiamenti politici, economici, sociali, ma soprattutto culturali abolendo le norme non scritte che rendono la donna cittadina di seconda classe.

Il WLUML ${ }^{62}$, Women Living Under Muslim Laws ${ }^{63}$ è un sito creato con lo scopo di monitorare la condizione femminile nel mondo islamico ed è composto sia da gruppi che da singoli individui. Il

\footnotetext{
${ }^{58}$ Ibidem, p. 17.

${ }^{59}$ Ibidem, p. 17.

${ }^{60}$ Ibidem, p. 18.

${ }^{61}$ ROBINSON, Julia, «Female Labor Force Participation in the Middle East and North Africa», in Wharton Research Scholars Journal, 28, 2005, pp. 1-46, p. 29, URL:

< http://repository.upenn.edu/cgi/viewcontent.cgi?article=1027\&context=wharton_research_scholars > [consultato il 19 novembre 2017].

${ }^{62}$ Women Living Under Muslim Laws (WLUML) è una rete di solidarietà internazionale che fornisce
} 
WLUML fornisce aiuto a tutte le donne musulmane che intendono contrastare leggi, istituzioni e costumi basati sulla disuguaglianza di genere, evidenziando l'uso politico della religione e invitando a far fronte comune contro le ingiustizie perpetrate in nome dell'Islam.

Non è il Corano a cambiare [...] sono le sue interpretazioni a essere mutate in relazione ai tempi e ai nuovi sentimenti di giustizia: il Corano professa la giustizia, l'uguaglianza e la fratellanza tra tutti gli esseri umani, bisogna creare nella propria epoca il modo più corretto per attuare questi principi ${ }^{64}$.

informazioni, sostegno e uno spazio collettivo per le donne le cui vite sono modellate, condizionate o governate da leggi e costumi che derivano dall'Islam, URL: <http://www.wluml.org/node/5408> [consultato il 19 novembre 2017].

${ }^{63}$ PEPICELLI, Renata, Femminismo islamico. Corano, diritti, riforme, cit., p. 85.

${ }^{64}$ Ibidem, p. 92. 


\section{L'AUTORE}

Arianna COLELLA ha conseguito la Laurea triennale in Storia presso l'Università di Bologna discutendo una tesi dal titolo Le conseguenze del golpe cileno del 1973 nella politica italiana degli anni '70. Attualmente frequenta il corso di Laurea Magistrale in Scienze storiche ed Orientalistiche, Curriculum Studi orientali, presso il medesimo ateneo.

URL: < http://www.studistorici.com/progett/autori/\#Colella > 